\title{
Talaromyces peaticola (Aspergillaceae, Eurotiales), a new species from the Zoige wetlands, China
}

\author{
Tian $\mathrm{JQ}^{1,2}$, Wang $\mathrm{YF}^{3}$ and Sun $\mathrm{JZ}^{1^{*}}$
}

${ }^{1}$ State Key Laboratory of Mycology, Institute of Microbiology, Chinese Academy of Sciences, No. 3 Park 1, Beichen West Road, Chaoyang District, Beijing 100101, P.R. China

${ }^{2}$ State Key Laboratory of Vegetation and Environmental Change, Institute of Botany, Chinese Academy of Sciences, Beijing 100093, China

${ }^{3}$ University of Chinese Academy of Sciences, 100049 Beijing, China

Tian JQ, Wang YF, Sun JZ 2021 - Talaromyces peaticola (Aspergillaceae, Eurotiales), a new species from the Zoige wetlands, China. Studies in Fungi 6(1), 391-399, Doi 10.5943/sif/6/1/29

\begin{abstract}
Species in Talaromyces section Trachyspermi are isolated from a wide range of substrates, including soil, house dust, leaf, wood and fruit from temperate region to tropical areas. During a survey of fungal diversity in Zoige wetlands, three isolates with biverticillate adpressed penicilli and spheroidal conidia belonging to Talaromyces are isolated from peat soil. Phylogenetic analyses based on a combined ITS, BenA, CaM and RPB2 sequence data suggests they represent a novel taxon in Talaromyces section Trachyspermi, namely Talaromyces peaticola. In spite of T. peaticola has a close affinity to $T$. diversus in phylogeny, it is readily distinguished from the later, resulted from growing slowly on CREA at $25^{\circ} \mathrm{C}$, exudating small clear droplets on MEA, and producing smaller conidia than that of $T$. diversus.
\end{abstract}

Keywords - 1 new taxon - peatland - Qinghai-Tibetan Plateau - taxonomy

\section{Introduction}

The genus Talaromyces was initially described by Benjamin (1955) to accommodate sexual morph Penicillium species with soft and yellowish ascomata surrounded by multiple layers of interwoven hyphae. Samson et al. (2011) redefined Talaromyces by transferring Penicillium subgenus Biverticillium into Talaromyces regarding the phylogenetic analysis of sequence data from the nuclear ribosomal internal transcribed spacer (ITS) and DNA-dependent RNA polymerase II largest subunit (RBP2) genes. Based on a multi-gene phylogeny of a combination of ITS, $\beta$ tubulin gene (BenA) and DNA-dependent RNA polymerase II second largest subunit (RPB2), Talaromyces has been divided into seven sections, namely as sections Bacillispori, Helici, Islandici, Purpurei, Subinflati, Talaromyces and Trachyspermi (Yilmaz et al. 2014). Currently, phylogenetic analyses of the ITS, BenA, CaM, and RPB2 genes are imperative for new species identification of Talaromyces (Yilmaz et al. 2014, Chen et al. 2016, Houbraken et al. 2020). The number of species in Talaromyces grew rapidly and have now reached more than 170 species (Houbraken et al. 2020).

Species in section Trachyspermi differ from other Talaromyces by conidiophores producing biverticillate phialides and when ascomata produced, have a creamy white or yellow color (Yilmaz et al. 2014, Chen et al. 2016, Wang et al. 2017). Additionally, they grow restrictedly on Czapek yeast autolysate agar (CYA), yeast extract sucrose agar (YES), and dichloran $18 \%$ glycerol agar 
(DG18), and slightly faster on malt extract agar (MEA) (Yilmaz et al. 2014, Chen et al. 2016, Luo et al. 2016, Romero et al. 2016, Wang et al. 2017). Consideration of the phylogeny and morphological features, 27species in Trachyspermi have been accepted by Houbraken et al. (2020) These species are always isolated from a wide range of substrates, including soil, house dust, leaf, wood and fruit from temperate region to tropical area (Chen et al. 2016, Romero et al. 2016, Wang et al. 2016). Their surviving strategy in the low osmotic environment, such as heat and dryresistance as well, and bioactive compounds were studied comprehensively (Frisvad et al. 2013, Chen et al. 2016, Romero et al. 2016).

Peatlands only cover about $3 \%$ of the land surface but currently maintain one-third of global carbon stores (Turetsky et al. 2015). Fungi, with their heterogeneous physiology, metabolic activities, and ecological functions, are recognized as key decomposers of organic matter in these ecosystems (Thormann et al. 2001, Gilbert \& Mitchell 2006). Studies of fungi in peatlands mostly focused on the relationship between fungal diversity and environmental factors using sequencing methods (Myers et al. 2012, Asemaninejad et al. 2017). A few culture-dependent studies indicated that Aspergillaceae (accounted for 25-30\%) are the most frequently isolated fungi from peatlands (Thormann 2006, Wu et al. 2013), while Talaromyces accounted for $4.2 \%$ of the isolates (Wu et al. 2013).

During a survey of fungal diversity in Zoige wetlands, three isolates with biverticillate adpressed penicilli and spheroidal rough walled conidia are isolated from peat soil. They are described here as a new taxon of Talaromyces based on single and combined ITS, BenA, CaM and the RPB2 gene sequences and cultural features on the recommended media.

\section{Materials \& methods}

\section{Soil collections and fungal isolation}

The cultures are isolated by dilution methods from soil samples collected in Zoige wetlands $\left(33^{\circ} 3^{\prime} 54 \mathrm{~N}, 102^{\circ} 34^{\prime} 23.9 \mathrm{E}\right)$ in Qinghai-Tibetan Plateau, China. Dried cultures are deposited in the Herbarium Mycologicum Academiae Sinicae (HMAS), and the ex-type strains are preserved in the China General Microbiological Culture Collection Center (CGMCC, http://www.cgmcc.net/english/OrderingOfCultures.html).

\section{Morphological observations and growth rate}

Macroscopic characters are studied on CYA, CYA supplemented with $5 \% \mathrm{NaCl}$ (CYAS), YES, creatine sucrose agar (CREA), DG18, oatmeal agar (OA) and MEA (Samson et al. 2011). Isolates are inoculated at three points on $90 \mathrm{~mm}$ Petri dishes and incubated for $7 \mathrm{~d}$ at $25^{\circ} \mathrm{C}$ in darkness. Additionally, CYA plates are incubated at 30 and $37^{\circ} \mathrm{C}$, and MEA plates were incubated at $30^{\circ} \mathrm{C}$. After $7 \mathrm{~d}$ of incubation, colony diameters are recorded. The colony texture, degree of sporulation, front and reverse colony colors, the production of soluble pigments and exudates are observed. Acid production on CREA is indicated by a change in the $\mathrm{pH}$ sensitive bromocresol purple dye, from a purple to yellow color in media surrounding colonies. Microscope preparations are made from 1-week old colonies grown on MEA (Yilmaz et al. 2014). A Nikon Ellipse 80i light microscope equipped with differential interference contrast (DIC) optics is used to capture digital images.

\section{DNA extraction, PCR amplification, and sequencing}

Isolates are grown on potato dextrose agar (PDA, Oxoid malt) 1 week, and fungal mycelium was scraped off for genomic DNA extraction. Genomic DNA is extracted by using a simple and rapid "thermolysis" method (Zhang et al. 2010) and stored at $-20^{\circ} \mathrm{C}$. The ITS, BenA, CaM, and RPB2 genes are amplified and sequenced using methods and primers previously described in Yilmaz et al. (2014). 
Table 1 Species used in phylogenetic analyses

\begin{tabular}{|c|c|c|c|c|c|}
\hline \multirow{2}{*}{ Species name } & \multirow{2}{*}{ Collection number ${ }^{a}$} & \multicolumn{4}{|c|}{ Accession number } \\
\hline & & BenA & CaM & ITS & RPB2 \\
\hline T. aerius & CBS $140611^{\mathrm{T}}$ & KU866835 & KU866731 & KU866647 & KU866991 \\
\hline T. albobiverticillius & CBS 133440T & KF114778 & KJ885258 & HQ605705 & KM023310 \\
\hline T. albobiverticillius & CBS 133441 & KF114777 & - & - & - \\
\hline T. assiutensis & CBS $147.78^{\mathrm{T}}$ & KJ865720 & KJ885260 & JN899323 & KM023305 \\
\hline T. assiutensis & CBS 645.80 & KF114802 & - & JN899334 & - \\
\hline T. assiutensis & CBS 116554 & KM066124 & - & KM066167 & \\
\hline T. atroroseus & CBS $133442^{\mathrm{T}}$ & KF114789 & KJ775418 & KF114747 & KM023288 \\
\hline T. atroroseus & DTO 270-D5 & KJ775227 & - & KJ775734 & - \\
\hline T. atroroseus & DTO 270-D6 & KJ775228 & - & KJ775735 & - \\
\hline T. austrocalifornicus & CBS $644.95^{\mathrm{T}}$ & KJ865732 & KJ885261 & JN899357 & - \\
\hline T. convolutus & CBS $100537^{\mathrm{T}}$ & KF114773 & - & JN899330 & JN121414 \\
\hline T. diversus & CBS $320.48^{\mathrm{T}}$ & KJ865723 & KJ885268 & KJ865740 & KM023285 \\
\hline T. diversus & DTO 131-I6 & KJ775193 & - & KJ775700 & - \\
\hline T. diversus & DTO 133-A7 & KJ775194 & - & KJ775701 & - \\
\hline T. erythromellis & CBS $644.80^{\mathrm{T}}$ & HQ156945 & KJ885270 & JN899383 & KM023290 \\
\hline T. heiheensis & HMAS $248789^{\mathrm{T}}$ & KX447525 & KX447532 & KX447526 & KX447529 \\
\hline T. minioluteus & CBS $642.68^{\mathrm{T}}$ & KF114799 & KJ885273 & JN899346 & JF417443 \\
\hline T. minioluteus & CBS 137.84 & KF114798 & - & KM066171 & - \\
\hline T. minioluteus & CBS 270.35 & KM066129 & - & KM066172 & - \\
\hline T. peaticola & CGMCC $3.18620^{\mathrm{T}}$ & MF284705 & MF284703 & MF135613 & MF284704 \\
\hline T. peaticola & CGMCC3.18767 & MF960859 & MF960861 & MF960857 & MF960863 \\
\hline T. peaticola & CGMCC3.18768 & MF960860 & MF960862 & MF960858 & MF960864 \\
\hline T. purpurogenus & CBS $286.36^{\mathrm{T}}$ & JX315639 & KF741947 & JN899372 & JX315709 \\
\hline T. rubrifaciens & CGMCC $3.17658^{\mathrm{T}}$ & KR855649 & KR855653 & KR855658 & KR855663 \\
\hline T. solicola & CBS $133445^{\mathrm{T}}$ & GU385731 & KJ885279 & FJ160264 & KM023295 \\
\hline T. solicola & CBS 133446 & KF114775 & - & KF114730 & - \\
\hline T. systylus & BAFCcult $3419^{\mathrm{T}}$ & KR233838 & KR233837 & КР026917 & - \\
\hline T. trachyspermus & CBS 118438 & KM066128 & - & КM066166 & - \\
\hline T. trachyspermus & CBS 116556 & KM066126 & - & KM066170 & - \\
\hline T. trachyspermus & CBS $373.48^{\mathrm{T}}$ & KF114803 & KJ885281 & JN899354 & JF417432 \\
\hline T. ucrainicus & CBS $162.67^{\mathrm{T}}$ & KF114771 & KJ885282 & JN899394 & KM023289 \\
\hline T. ucrainicus & CBS 127.64 & - & - & KM066173 & - \\
\hline T. ucrainicus & CBS 583.72A & KM066130 & - & KM066174 & - \\
\hline T. udagawae & CBS $579.72^{\mathrm{T}}$ & KF114796 & KX961260 & JN899350 & - \\
\hline
\end{tabular}

\section{Phylogenetic analysis}

The ITS sequences of isolates are firstly blasted on NCBI, resulting in $99 \%$ similar to Trachyspermi diversus ex-type strain CBS 320.48, which belongs to Talaromyces section Trachyspermi. Then, sequences of ITS, BenA, CaM, and RPB2 of species belong to section Trachyspermi determined from recent studies (Yilmaz et al. 2014, Chen et al. 2016, Luo et al. 2016, Romero et al. 2016, Wang et al. 2016, 2017, Houbraken et al. 2020), are downloaded from GenBank (Table 1). A single gene data-set is aligned using MAFFT version 7.03 with the Q-INS-I strategy (Katoh \& Standley 2013). The ambiguous areas of alignment are located and removed using the Gblocks version 0.91b software program (Castresana 2000). The appropriate nucleotide substitution model for each gene is tested using the Akaike information criterion (AIC) with MrModeltest v2.3 (Nylander 2004). The 'GTRGAMMAI' model is the best model for ITS, BenA and RPB2 sequences, and the 'GTRGAMMA' model is the best model for CaM sequences. The model for multi-gene analysis is the combination of all models of the single gene. Talaromyces purpurogenus in Talaromyces section Talaromyces is set as outgroup in each analysis.

Combined sequences of the ITS, BenA, CaM, and RPB2 are concatenated using the Sequence Matrix for Windows version 1.7.8 (Vaidya et al. 2011), and missing data are treated as gaps. Single and combined genes are analyzed using maximum likelihood (ML) performed in RAxML (Stamatakis 2006) implemented in raxmlGUI v.1.3 (Silvestro \& Michalak 2012) with rapid 
bootstrap analysis with 1000 replicates. For Bayesian analyses, the posterior probabilities were determined by Markov chain Monte Carlo sampling (MCMC) in MrBayes v3.2 (Ronquist et al. 2012) based on the best models from MrModeltest. An average standard deviation of $<0.01$ for split frequencies is used to suggest a convergence between parallel runs. The first $25 \%$ of sampled trees were discarded as 'burn-in'. Trees are figured in FigTree v1.4.2 (Rambaut 2014). Bootstrap values higher than 70\% from Paup (BSMP), RAxML (BSML), and Bayesian posterior probabilities (BYPP) greater than 0.95 are indicated in the phylogenetic trees.

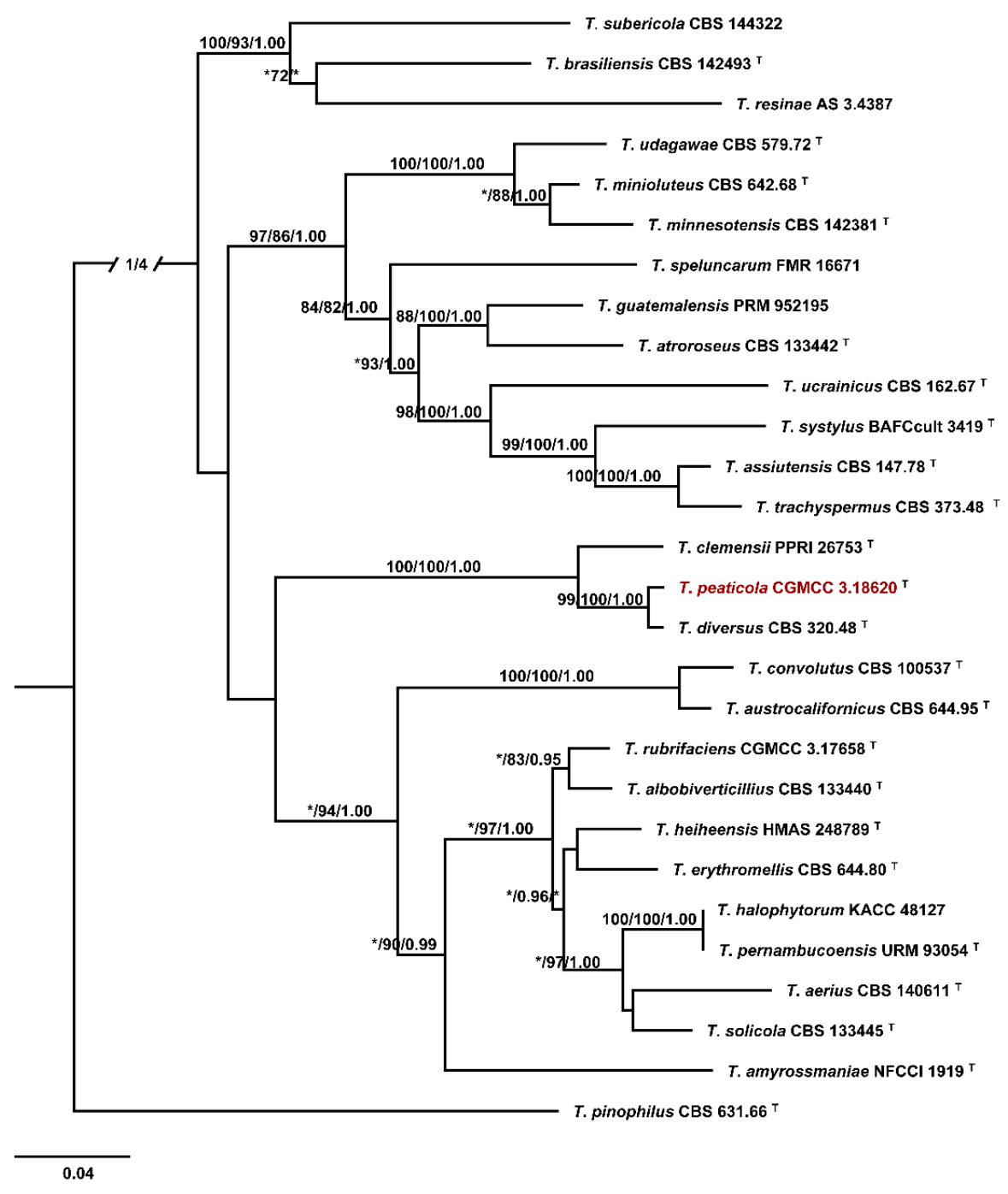

Fig. 1 - Phylogenetic tree generated from ML analysis combined ITS, BenA, CAM and RPB2 sequence data for Talaromyces section Trachyspermi. Talaromyces purpurogenus was chosen as outgroup. Bootstrap values higher than $70 \%$ for MP analysis (BSMP) (left) and ML analysis (BSML) (middle) are given above the nodes respectively. Bayesian posterior probabilities greater than 0.95 are indicated (BYPP) (right). * indicates bootstrap values of less than $70 \%$ or Bayesian posterior probabilities lower than 0.95 for a lineage. ${ }^{\mathrm{T}}$ indicates the ex-living type.

\section{Results}

\section{Phylogenetic analyses}

A first phylogeny concerning all currently accepted species in section Trachyspermi, including the type stain of our new isolates, was performed by using a sequence data-set of combined ITS (448 bp), BenA (351 bp), CaM (476 bp), and RPB2 (841 bp) genes (Fig. 1). The phylogenetic tree presented our putative new species Talaromyces peaticola (CGMCC 3.18620) 
positioned robustly in section Trachysperm. Within section, T. peaticola (CGMCC 3.18620), Talaromyces clemensii (PPRI 26753), and T. diversus (CBS 320.48) formed a clade with strong support (100, BSMP / 100, BSML / 1.00, BYPP). Within this clade, CGMCC 3.18620 and T. diversus (CBS 320.48) formed a distinct subclade with strong support (99, BSMP / 100, BSML / $1.00, \mathrm{BYPP})$ as well, suggesting CGMCC 3.18620 is closely related to $T$. diversus. Consideration of the limited resolution of the ITS in the Trichocomaceae, BenA was proposed as the secondary DNA barcode for Talaromyces (Yilmaz et al. 2014). A phylogenetic tree was reconstructed by employing all available sequences BenA of species in section Trachysperm (Fig. 2). It presented that all isolates of $T$. peaticola and $T$. diversus formed a clade with strong support (100, BSMP / 100, BSML / 1.00, BYPP). Within this clade, T. peaticola formed a distinct subclade with strong support (95, BSMP / 75, BSML / 1.00, BYPP) separated from T. diversus, suggesting T. diversus is new taxon.

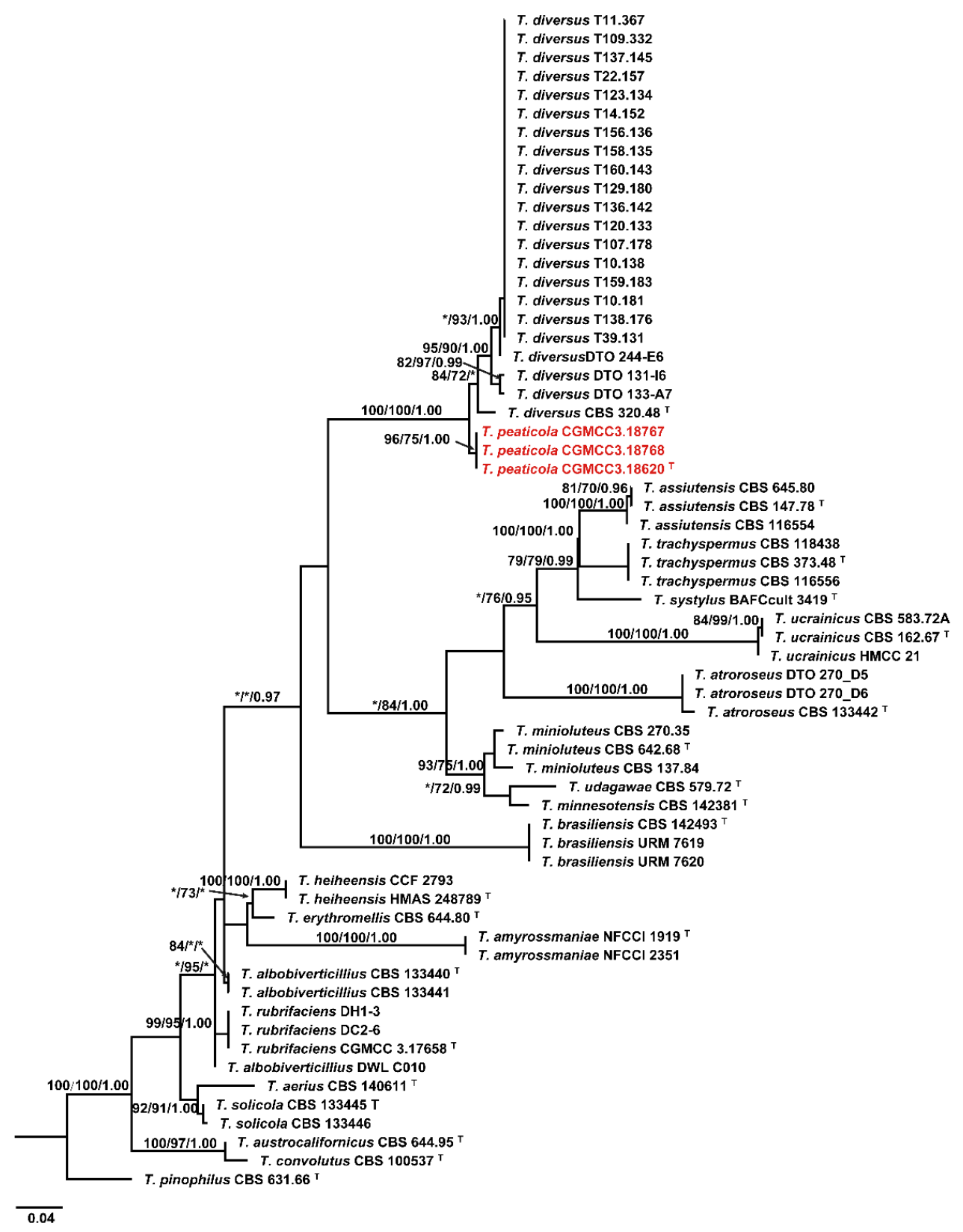

Fig. 2 - Phylogenetic tree generated from ML analysis BenA sequence data for Talaromyces section Trachyspermi. Talaromyces purpurogenus was chosen as an outgroup. Bootstrap values higher than 70\% for MP analysis (BSMP) (left) and ML analysis (BSML) (middle) are given above 
the nodes respectively. Bayesian posterior probabilities greater than 0.95 are indicated (BYPP) (right). * indicates bootstrap values of less than $70 \%$ or Bayesian posterior probabilities lower than 0.95 for a lineage. ${ }^{\mathrm{T}}$ indicates the ex-living type.

Talaromyces peaticola Jian Q. Tian \& Jing Z. Sun sp. nov.

Fig. 3

Index Fungorum number: IF553909; Facesoffungi number: FoF 09706

Etymology - peaticola, the stem peati- refers to the substrate that the type strain is isolated, the ending -cola means"dweller, inhabit".

Diagnosis - Colonies slow-growing and concave in centers on CYA at $25^{\circ} \mathrm{C}$, extremely slowgrowing on $\mathrm{CYA}$ at $30^{\circ} \mathrm{C}$ and $37^{\circ} \mathrm{C}$, acid production absents on CREA at $25^{\circ} \mathrm{C}$; conidiophores biverticillate; conidia globose, smooth-walled.
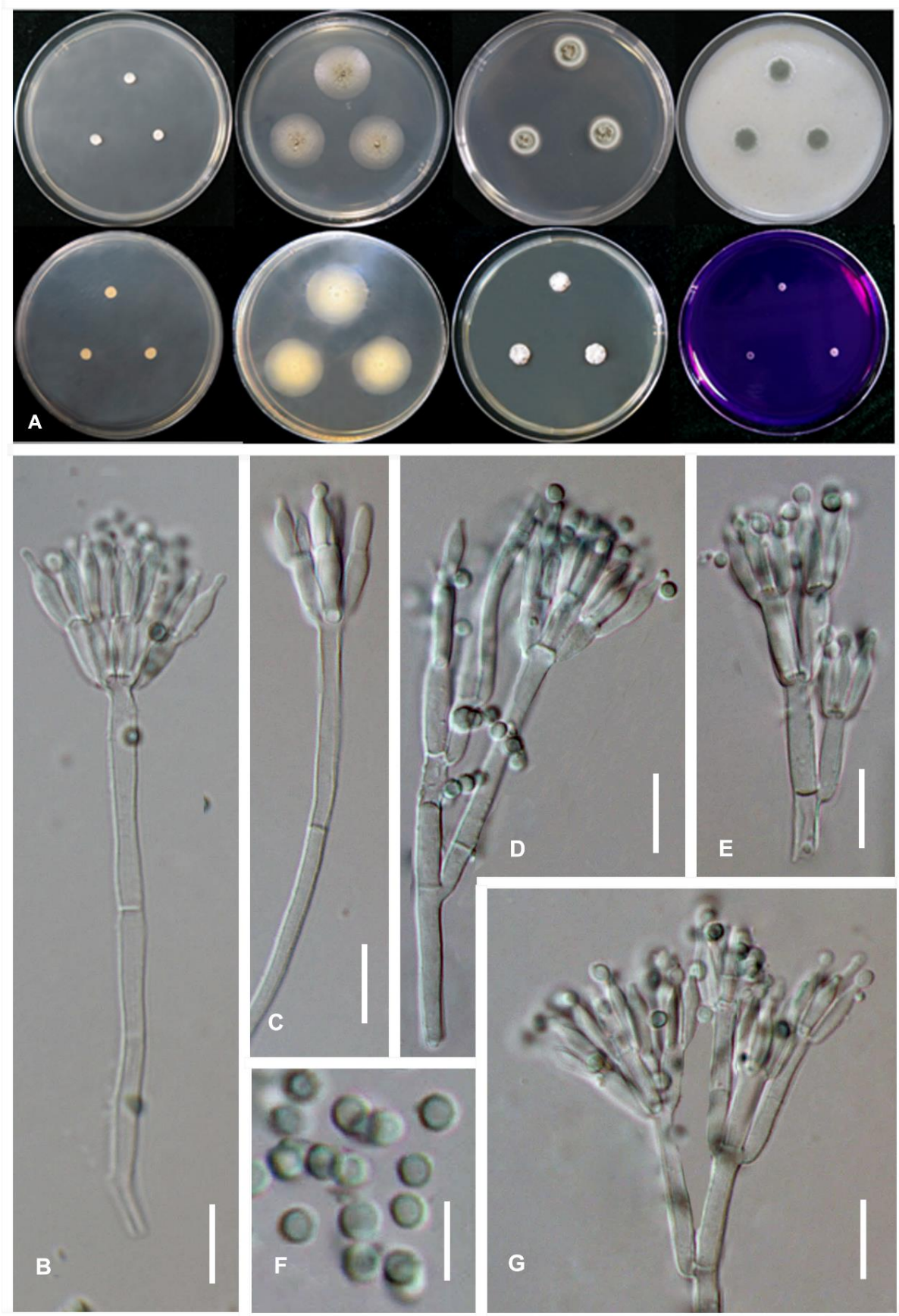

Fig. 3 - Morphological characters of T. peaticola (ex-type CGMCC3.18620). A Colonies from left to right (top row) CYA, MEA, YES, and OA; (bottom row) CYA reverse, MEA reverse, DG 18 and CREA. B-G Conidiophores. H Conidia. Scale bars: B-C $=20 \mu \mathrm{m}, \mathrm{D}-\mathrm{H}=10 \mu \mathrm{m}$. 
Colony diam, $7 \mathrm{~d}, 25^{\circ} \mathrm{C}$ (unless stated otherwise): CYA 5.0-6.9 mm; MEA 24.7-24.9 mm; DG18 16.0-16.5 mm; YES 9 -11 mm; CREA 3.3-3.9 mm; OA 11.7-12.5 mm; CYAS 2.9-3.5 mm; CYA $30^{\circ} \mathrm{C} 4.7-6.6 \mathrm{~mm}$; CYA $37^{\circ} \mathrm{C} 4.5-4.7 \mathrm{~mm}$; MEA $30^{\circ} \mathrm{C} 35.5-37.8 \mathrm{~mm}$.

Colony characters - CYA $25^{\circ} \mathrm{C}, 7 \mathrm{~d}$ : colonies slightly raised at center, slightly sulcate; mycelia white; texture velvety; sporulation absent; soluble pigments absent; exudates absent; reverse medium beige (Fig. $3 \mathrm{~A})$. MEA $25^{\circ} \mathrm{C}$, $7 \mathrm{~d}$ : colonies low plane; margins low, plane, entire; mycelia white; sporulation loose; conidia grayish green; texture floccose; soluble pigments absent; exudates small clear droplets; reverse bone white (Fig. 3A). YES $25^{\circ} \mathrm{C}, 7 \mathrm{~d}$ : colonies slightly raised at center, sunken at center, sulcate; margin slow, plane, entire $(<1 \mathrm{~mm})$; mycelia white; texture velvety; sporulation absents to sparse; soluble pigment absent; exudates absent; reverser white (Fig. $3 \mathrm{~A})$. DG18 $25^{\circ} \mathrm{C}, 7 \mathrm{~d}$ : colonies raised at center, sunken in the centre, sulcate; margins low, plane, entire (1-2 mm); mycelia white; texture velvety; sporulation moderately dense to dense; conidia dark green; soluble pigments absent; exudate absent (Fig. 3A). OA $25^{\circ} \mathrm{C}$, 7d: colonies low, plane; margins low, plane, entire; mycelia white; texture floccose; sporulation dense, conidia dark green; soluble pigments absent; exudates absent (Fig. 3A); CREA $25^{\circ} \mathrm{C}$, $7 \mathrm{~d}$ : restricted growth; acid production absent (Fig. 3A). On MEA $25^{\circ} \mathrm{C}$, $7 \mathrm{~d}$ conidiophores biverticillate (Fig. 3B-G); stipes smooth-walled, 160-200 × 3-4 $\mu \mathrm{m}$ (Fig. 3D, G); metulae, 3-8, divergent, 7.5-11.5 × 2-3 $\mu \mathrm{m}$; phialides acerose, per metulae, 7.0-13.5 $\times 1.5-2.0 \mu \mathrm{m}$ (Fig. 3E); conidia smooth, in chain, subglobose, globose, smooth-walled, 1.5-2.5×1.5-2.0 $\mu \mathrm{m}$ (Fig. 3H).

Teleomorph - Undetermined

Known distribution - CHINA. Sichuan Province.

Material examined - CHINA. Sichuan Province, Aba Autonomous Prefecture, Hongyuan County, Zoigê wetland, $33^{\circ} 3^{\prime} 54 \mathrm{~N}, 102^{\circ} 34^{\prime} 23.9 \mathrm{E}$, peaty soil, September 15, 2016, Jianqing Tian, ZRT-4 (holotype: HMAS 247296). ex-type living culture: CGMCC3.18620, CGMCC3.18767, CGMCC3.18768. ibid. LB1.17020001.

Notes - Talaromyces peaticola belongs to Talaromyces section Trachyspermi are well supported by the phylogenetic analyses of a combination of ITS, BenA, CaM and RPB2 sequence data-set. BenA marker and multi-gene of ITS, BenA, CaM and RPB2 performed well in differing $T$. peaticola from $T$. diversus. It also can be distinguished from other Talaromyces species by colony slow-growing on CYA at $25^{\circ} \mathrm{C}$, extremely slow-growing on CYA at $30^{\circ} \mathrm{C}$ and $37^{\circ} \mathrm{C}$, acid production absents on CREA at $25^{\circ} \mathrm{C}$; conidiophores biverticillate; conidia globose, smooth-walled. In spite of $T$. peaticola phylogenetically and morphologically closed to $T$. diversus, it could be easily distinguished from $T$. diversus by the later could not grow on CREA at $25^{\circ} \mathrm{C}$ and $T$. peaticola producing smaller conidia (Yilmaz et al. 2014).

\section{Discussion}

The taxonomy of Talaromyces was redefined recently on the basis of DNA sequence data, extrolite profiles and other phenotypic features (Yilmaz et al. 2012, 2014, Chen et al. 2016). The phylogenetic analysis resulted from the combined sequence of ITS, BenA, CaM and RPB2 well distinguish Talaromyces peaticola from other Talaromyces species in section Trachyspermi (Fig. 1), which supported combined ITS, BenA, CaM and RPB2 sequence is imperative for new species identification (Yilmaz et al. 2014, Chen et al. 2016). Additionally, phylogenetic analysis conducted by the single gene of BenA well differs $T$. peaticola from other species within Talaromyces section Trachyspermi, as well as its sister taxon $T$. diversus, which resulted from a 9 bp difference in BenA locus (448/457). This confirms that BenA, is imperative for new species identification of Talaromyces (Yilmaz et al. 2014, Chen et al. 2016). However, both phylogenetic analyses based on the single gene of ITS and RPB2 not well differ $T$. peaticola from $T$. diversus owning the little differences ITS sequence (585/590 bp) and RPB2 sequences ( $850 / 852 \mathrm{bp})$. These results agree with that ITS and RPB2 sequences are insufficiently variable to reliably discriminate species in Talaromyces (Skouboe et al. 1999, Yilmaz et al. 2012, Frisvad et al. 2013, Yilmaz et al. 2014). BenA and CaM sequences perform well in species delimitation of Aspergillaceae, especially in 
Penicillium, Aspergillus and Talaromyces, and even some intraspecies (Seifert et al. 2007, Visagie et al. 2014, Yilmaz et al. 2014, Chen et al. 2016).

Colony features on seven standardized media and morphological characters on MEA and are recommended as important phenotypic features in the identification of Talaromyces (Visagie et al. 2014, Yilmaz et al. 2014). Talaromyces peaticola characteristically displays restricted growth on CYA and CREA and exudates small clear droplets on MEA (Fig. 3). In spite that T. peaticola has an affinity to $T$. diversus in all phylogenetic trees (Fig. 1). These two species are readily distinguished from each otherin regard to $T$. diversus could not grow on CREA at $25^{\circ} \mathrm{C}$ and exudates small clear droplets on MEA (Yilmaz et al. 2014). Additionally, the conidia of $T$. peaticola are smaller than of $T$. diversus. Therefore, Talaromyces peaticola is introduced as a new taxon.

Zoige peatland is the largest alpine peatland, characterized by high moisture and low temperature. Previous studies have reported that species in genus Talaromyces are one of the major fungal survivals and frequently isolated from peatland (Gilbert \& Mitchell 2006, Wu et al. 2013, Grum-Grzhimaylo et al. 2016, Asemaninejad et al. 2017), the ecological function and metabolic activities need to be further explored.

\section{Acknowledgements}

This research is jointly supported by the National Key Research and Development Program of China (No. 2016YFC0501802) and the Natural Science Foundation of China (no. 31600024).

\section{References}

Asemaninejad A, Thorn RG, Lindo Z. 2017 - Vertical distribution of fungi in hollows and hummocks of boreal peatlands. Fungal Ecology 27, 59-68.

Benjamin CR. 1955 - Ascocarps of Aspergillus and Penicillium. Mycologia 47, 669-687.

Castresana J. 2000 - Selection of conserved blocks from multiple alignments for their use in phylogenetic analysis. Molecular Biology and Evolution 17, 540-552.

Chen AJ, Sun BD, Houbraken J, Frisvad JC et al. 2016 - New Talaromyces species from indoor environments in China. Studies in Mycology 84, 119-144.

Frisvad JC, Yilmaz N, Thrane U, Rasmussen KB et al. 2013 - Talaromyces atroroseus, a new species efficiently producing industrially relevant red pigments. Plos One 8. Doi 10.1371/journal.pone.0084102

Gilbert D, Mitchell EA. 2006 - Microbial diversity in Sphagnum peatlands. Developments in Earth Surface Processes 9, 287-318.

Grum-Grzhimaylo OA, Debets AJM, Bilanenko EN. 2016 - The diversity of microfungi in peatlands originated from the White Sea. Mycologia 108, 233-254.

Houbraken J, Kocsubé S, Visagie CM, Yilmaz N et al. 2020 - Classification of Aspergillus, Penicillium, Talaromyces and related genera (Eurotiales): An overview of families, genera, subgenera, sections, series and species, Studies in Mycology 95, 5-169.

Katoh K, Standley DM. 2013 - MAFFT Multiple sequence alignment software version 7: improvements in performance and usability. Molecular Biology and Evolution 30, 772-780.

Luo Y, Lu XH, Bi W, Liu F, Gao WW. 2016 - Talaromyces rubrifaciens, a new species discovered from heating, ventilation and air conditioning systems in China. Mycologia 108, 773-779.

Myers B, Webster KL, Mclaughlin JW, Basiliko N. 2012 - Microbial activity across a boreal peatland nutrient gradient: the role of fungi and bacteria. Wetlands Ecology and Management 20, 77-88.

Nylander JAA. 2004 - MrModeltest v2. Program distributed by the author. Available from: http://www.abc.se/ nylander/ mrmodeltest2/mrmodeltest2.html

Rambaut A. 2014 - FigTree v1. 4.2 from http://tree.bio.ed.ac.uk/software/figtree/ (Retrieved on July 1, 2016) 
Romero SM, Romero AI, Barrera V, Comerio R. 2016 - Talaromyces systylus, a new synnematous species from Argentinean semi-arid soil. Nova Hedwigia 12, 241-256.

Ronquist F, Teslenko M, van der Mark P, Ayres DL et al. 2012 - MrBayes 3.2: Efficient Bayesian phylogenetic inference and model choice across a large model space. Systematic Biology 61, 539-542.

Samson RA, Yilmaz N, Houbraken J, Spierenburg H et al. 2011 - Phylogeny and nomenclature of the genus Talaromyces and taxa accommodated in Penicillium subgenus Biverticillium. Studies in Mycology 70, 159-183.

Seifert KA, Samson RA, Houbraken J, Lévesque CA et al. 2007 - Prospects for fungus identification using CO1 DNA barcodes, with Penicillium as a test case. Proceedings of the National Academy of Sciences 104, 3901-3906.

Silvestro D, Michalak I. 2012 - raxmlGUI: a graphical front-end for RAxML. Organisms Diversity \& Evolution 12: 335-337.

Skouboe P, Frisvad JC, Taylor JW, Lauritsen D et al. 1999 - Phylogenetic analysis of nucleotide sequences from the ITS region of terverticillate Penicillium species. Mycological Research $103,873-881$.

Stamatakis A. 2006 - RAxML-VI-HPC: Maximum likelihood-based phylogenetic analyses with thousands of taxa and mixed models. Bioinformatics 22, 2688-2690.

Thormann MN. 2006 - Diversity and function of fungi in peatlands: A carbon cycling perspective. Canadian Journal of Soil Science 86, 281-293.

Thormann MN, Bayley SE. Currah RS. 2001 - The microbial wildcard in peatland carbon storage: implications for global warming. Proceedings of the International Workshop on Carbon Dynamics of Forested Peatlands: Knowledge Gaps, Uncertainty and Modeling Approaches.

Turetsky MR, Benscoter B, Page S, Rein G et al. 2015 - Global vulnerability of peatlands to fire and carbon loss. Nature Geoscience 8, 11-14.

Vaidya G, Lohman DJ, Meier R. 2011 - SequenceMatrix: concatenation software for the fast assembly of multi-gene datasets with character set and codon information. Cladistics 27 , 171-180.

Visagie CM, Hirooka Y, Tanney JB, Whitfield E et al. 2014 - Aspergillus, Penicillium and Talaromyces isolated from house dust samples collected around the world. Studies in Mycology 78, 63-139.

Wang XC, Chen K, Qin WT, Zhuang WY. 2017 - Talaromyces heiheensis and T. mangshanicus, two new species from China. Mycological Progress 16, 73-81.

Wang XC, Chen K, Xia YW, Wang L et al. 2016 - A new species of Talaromyces (Trichocomaceae) from the Xisha Islands, Hainan, China. Phytotaxa 267, 187-200.

Wu B, Tian JQ, Bai CM, Xiang MC et al. 2013 - The biogeography of fungal communities in wetland sediments along the Changjiang River and other sites in China. ISME Journal 7(7), 1299-1309.

Yilmaz N, Houbraken J, Hoekstra ES, Frisvad JC, Visagie1 CM, Samson RA. 2012 - Delimitation and characterisation of Talaromyces purpurogenus and related species. Persoonia 29, 39-54.

Yilmaz N, Visagie J, Houbraken, CM, Frisvad JC, Samson RA. 2014 -Polyphasic taxonomy of the genus Talaromyces. Studies in Mycology 78, 175-341.

Zhang YJ, Zhang S, Liu XZ, Wen HA, Wang M. 2010 - A simple method of genomic DNA extraction suitable for analysis of bulk fungal strains. Letters in Applied Microbiology 51, 114-118. 